\title{
Perceptions of the barriers, facilitators, outcomes, and helpfulness of strategies to implement screening, brief intervention, and referral to treatment in acute care
}

\author{
Alyson Keen ${ }^{1,2^{*}}$, Kelli Thoele ${ }^{1}$, Ukamaka Oruche ${ }^{1}$ and Robin Newhouse ${ }^{1}$
}

\begin{abstract}
Background: Screening, Brief Intervention, and Referral to Treatment (SBIRT) is a clinical intervention used to address alcohol and illicit drug use. SBIRT use has resulted in positive health and social outcomes; however, SBIRT implementation remains low. Research on implementing interventions, such as SBIRT, lacks information about challenges and successes related to implementation. The Expert Recommendations for Implementing Change (ERIC) provides a framework to guide comprehension, clarity, and relevance of strategies available for implementation research. This framework was applied to qualitative feedback gathered from site coordinators (SCs) leading SBIRT implementation. The purpose of this study was to describe the SCs' experiences pertaining to SBIRT implementation across a health system.
\end{abstract}

Methods: Within the context of a larger parent study, a semi-structured interview guide was used to capture 14 SCs' perceptions of the barriers, facilitators, and outcomes pertaining to SBIRT implementation. Qualitative data were analyzed using standard content analytic procedures. A follow-up survey was developed based on 14 strategies identified from qualitative data and was administered electronically to determine the SC's perceptions of the most helpful implementation strategies on a scale of 1 (least helpful) to 5 (most helpful).

Results: All 14 invited SCS participated in the SBIRT implementation interview, and 11 of 14 (79\%) responded to the follow-up survey. Within the categories of barriers, facilitators, and outcomes, 25 subthemes emerged. The most helpful implementation strategies were reexamining the implementation $(M=4.38 ; n=8)$, providing ongoing consultation $(M=4.13 ; n=8)$, auditing and providing feedback $(M=4.1 ; n=10)$, developing education materials $(M=4.1 ; n=10)$, identifying and preparing champions $(M=4 ; n=7)$, and tailoring strategies $(M=4 ; n=7)$.

\footnotetext{
* Correspondence: akeen2@iuhealth.org

${ }^{1}$ Indiana University School of Nursing, 600 Barnhill Dr., Indianapolis, IN 46202,

USA

${ }^{2}$ Indiana University Health Adult Academic Health Center, 1701 N. Senate

Ave, Indianapolis, IN 46202, USA
}

(c) The Author(s). 2021 Open Access This article is licensed under a Creative Commons Attribution 4.0 International License, which permits use, sharing, adaptation, distribution and reproduction in any medium or format, as long as you give appropriate credit to the original author(s) and the source, provide a link to the Creative Commons licence, and indicate if changes were made. The images or other third party material in this article are included in the article's Creative Commons licence, unless indicated otherwise in a credit line to the material. If material is not included in the article's Creative Commons licence and your intended use is not permitted by statutory regulation or exceeds the permitted use, you will need to obtain permission directly from the copyright holder. To view a copy of this licence, visit http://creativecommons.org/licenses/by/4.0/ The Creative Commons Public Domain Dedication waiver (http://creativecommons.org/publicdomain/zero/1.0/) applies to the data made available in this article, unless otherwise stated in a credit line to the data. 
(Continued from previous page)

Conclusion: SCs who led implementation efforts within a large healthcare system identified several barriers and facilitators to the implementation of SBIRT. Additionally, they identified clinician-related outcomes associated with SBIRT implementation into practice as well as strategies that were helpful in the implementation process. This information can inform the implementation of SBIRT and other interventions in acute care settings.

Keywords: Screening, Brief Intervention, Referral to Treatment (SBIRT); Addiction; Substance-related disorders, Implementation, Strategies

\section{Contributions to the literature}

- This study examined site coordinators' (SCS) perceptions of implementing a clinical intervention by applying the Expert Recommendations for Implementing Change (ERIC) framework to identify commonly used and helpful implementation strategies.

- Study findings highlight barriers (e.g., sustainment, negative attitudes), facilitators (e.g., leveraging interdisciplinary support, adapting intervention to organizational context), and outcomes (e.g., increased awareness, action-oriented approach) perceived by SCs while implementing SBIRT, such findings can be considered by other change leaders preparing for intervention implementation.

- Considering there are 73 strategies in the ERIC framework, identifying implementation strategies most frequently used and most helpful (e.g., purposely reexamine the implementation, provide ongoing consultation) will help both researchers and clinicians prioritize strategies for future implementation projects or research.

- While this study identified the perceived helpfulness of multiple implementation strategies, future studies are needed to understand the mechanisms of action for specific implementation strategies deemed helpful to change leaders.

\section{Background}

The addiction epidemic has plagued the USA, with 20.3 million Americans affected and an annual economic burden of $\$ 740$ billion $[1,2]$. Health-related consequences of addiction range from appetite, sleep, and mood changes to heart attack, stroke, overdose, and death [3]. Approximately 70,237 overdose deaths resulted from addiction in the USA in 2017 [4]. Of the 21.2 million Americans needing treatment in 2018, only $11.1 \%$ received specialty addiction treatment [1].

Screening, Brief Intervention, and Referral to Treatment (SBIRT) is endorsed by the Substance Abuse and Mental Health Services Administration [5] as an intervention to address alcohol and illicit drug use [6]. SBIRT has been implemented in multiple healthcare settings [7], including acute care, where patients are typically seeking care for health issues unrelated to addiction. In particular, SBIRT implementation on medical surgical units is associated with a decrease in risky alcohol use $[8,9]$. Although SBIRT is associated with positive health and social outcomes [10-12] as well as positive clinician outcomes (e.g., increased SBIRT skills, improved selfefficacy) $[5,13]$, SBIRT implementation remains low [11, 12].

Despite a growing understanding of how and why interventions are adopted, implemented, and sustained, the concepts and descriptions of implementation strategies have been inconsistent in the literature. The Expert Recommendations for Implementing Change (ERIC) definitions by Powell et al. provides a framework to organize implementation strategies and inform comprehension, clarity, and relevance of 73 strategies to be considered as options for use for implementation research [14]. Commonly referenced strategies include tailored implementation strategies, educational outreach, printed education materials, local champion leaders, educational meetings, audit and feedback, and computerized reminders. However, more work is needed to clearly identify strategies and their associated mechanism of action that facilitate and enhance implementation from the lens of clinicians implementing change [15].

To begin to fill this gap, qualitative interview data were gathered from local champions (i.e., site coordinators) leading SBIRT implementation (i.e., parent study) in a large health care system in a Midwestern state in the USA. Responses were categorized into the implementation strategies described by Powell et al. [14]. Findings will enhance implementation scientists' understanding of strategies commonly used and perceived by clinicians (nurses in this case) as most helpful for implementing SBIRT in acute care settings.

The parent study was completed using a phased cluster randomized design to evaluate nurse-led SBIRT [16]. In this parent study, participating hospitals selected a nurse site coordinator (SC) at each facility championing SBIRT implementation and complete required study training. The 8-h training included content and 
competency validation on SBIRT and implementation strategies, as well as a discussion of health system change [17]. SCs at each hospital tailored the SBIRT intervention to their clinical site by addressing barriers identified in their baseline and implementation assessment and adapting strategies based on the unit context [18]. During monthly study team calls, various challenges and successes emerged from observations and feedback from the SCs. The purpose of this study was to describe the SCs' experiences implementing SBIRT across the healthcare system. Specific aims were to describe SCs' perceptions of (1) barriers, facilitators, and outcomes related to SBIRT implementation; and (2) strategies perceived as most helpful to implement SBIRT.

\section{Methods}

The parent study took place on 14 medical surgical units (i.e., one per hospital). Hospital settings included academic health centers, community hospitals, and critical access hospitals. Study participants were 14 study nurse SCs in various positions including direct care clinical nurses $(n=8)$, clinical educators $(n=2)$, clinical nurse specialists $(n=2)$, a nurse case manager $(n=1)$, and a house supervisor $(n=1)$. Within the context of the parent study, a semi-structured interview guide was used to capture the 14 SCs' experiences pertaining to barriers, facilitators, and outcomes of SBIRT implementation. The SQUIRE 2.0 Revised Standards for Quality Improvement Reporting Excellence were used for reporting [19].

\section{Data collection}

The interview guide was sent to all SCs for reflection prior to the interview and then data were collected via one-on-one telephone interviews with a research assistant or via e-mail. Responses to interview questions were recorded in a Word file on the semi-structured interview guide. Participants responded to the following interview questions: (1) what factors were the most helpful in the implementation of SBIRT?; (2) What barriers to implementation did you encounter?; (3) In what ways did study activities improve, help, or hinder care in your facility? Although interviews were not audio-recorded, notes were recorded in a Word file. The implementation strategies mentioned during the interviews were then categorized by the research assistant into the strategies described by Powell et al. [14], and a follow-up survey was created based on 14 commonly identified strategies described by SCs in the qualitative interviews. The survey was administered electronically and SCs responded by indicating the strategies that they used during the study and ranking the most helpful strategies on a scale from 1 (least helpful) to 5 (most helpful). The interviews and follow-up surveys were conducted during the 2 months (June and July 2019) following completion of the 12-month parent study.

\section{Data analysis}

Interview responses were compiled into an Excel spreadsheet. Responses were categorized into themes and subthemes using standard content analysis procedures by grouping similar responses [20]. The number of participant statements reflecting each theme/sub-theme was quantified by a research assistant (a PhD student). A second research assistant (also a PhD student) then independently reviewed the notes from the interviews and categorized the responses into themes and sub-themes. The two research assistants then reviewed the data together and verbally resolved all discrepancies. Further validation was completed with the SC group. SCs were provided the final list of themes and subthemes to evaluate content accuracy and potential missing content from the de-identified interview data. The response size and average rating for each implementation strategy were calculated. Implementation strategies were ordered from highest to lowest average rating to identify which strategies were considered most helpful to the SCs.

\section{Results}

\section{Interviews}

All 14 SCs provided qualitative data regarding SBIRT implementation. Although phone interviews were requested from all site coordinators, two site coordinators (14\%) preferred to send responses to the interview questions via email, while the remaining twelve (86\%) site coordinators completed the interviews via phone. Phone interviews ranged from 15 to $45 \mathrm{~min}$. Three major themes were pre-determined based on the aims of the study (implementation barriers, facilitators, and outcomes), and within these themes, 25 subthemes emerged. A detailed list of subthemes with examples is provided in Table 1 and the most common subthemes with quotes from SCs are provided below.

\section{Theme 1: implementation barriers}

Participants described several factors that hindered the timely and seamless implementation of SBIRT. The most common barriers were challenges sustaining the implementation of SBIRT, collecting data from the electronic medical record to evaluate implementation, and staff nurse resistance to the use of SBIRT due to negative attitudes regarding the intervention. While reflecting on barriers to implementation, one participant stated, "Sustainment was difficult because the process was on paper and not yet integrated into the electronic medical record. Our providers and nurses are still asking about substance use but not with a validated assessment tool." Another participant stated: 
Table 1 Site coordinator perception of SBIRT implementation

\begin{tabular}{|c|c|c|}
\hline Theme & Sub-theme (\# mentions) & Examples \\
\hline \multirow[t]{11}{*}{$\begin{array}{l}\text { Barriers identified related to SBIRT implementation } \\
\text { were: }\end{array}$} & Sustainment (10) & $\begin{array}{l}\text { Process complacency; difficult to coordinate with multiple } \\
\text { clinicians }\end{array}$ \\
\hline & Data collection (9) & Difficult to navigate health record; data absent from record \\
\hline & Staff adoption (9) & Nurse adherence to SBIRT; negative attitudes \\
\hline & Unit operational challenges (8) & Leader turnover; new nurses on the unit \\
\hline & SC execution (7) & SC felt alone in implementation efforts; SC role unclear \\
\hline & Study rollout (7) & Trial originally set up on different unit; site coordinator changed \\
\hline & Training coordination (6) & Finding a training schedule that worked for staff \\
\hline & Brief Intervention (BI) (5) & Lack of understanding of intent; discomfort with BI process \\
\hline & Patient-specific (4) & Patients did not see relevance or did not want help \\
\hline & Patient referral (2) & Insurance challenges; lack of available referral sites \\
\hline & Effort duplication (2) & $\begin{array}{l}\text { SBIRT activities/documentation duplicated (nursing and social } \\
\text { work) }\end{array}$ \\
\hline \multirow[t]{7}{*}{$\begin{array}{l}\text { Facilitators identified related to SBIRT implementation } \\
\text { were: }\end{array}$} & Leveraging support (9) & $\begin{array}{l}\text { Involvement of interprofessional stakeholders; use of early } \\
\text { adopters }\end{array}$ \\
\hline & Adapting intervention (8) & Ability to tailor/create resources; dedicated location for resources \\
\hline & SC development (8) & $\begin{array}{l}\text { Learning and peer support from other SC; research team } \\
\text { mentorship }\end{array}$ \\
\hline & Feedback loop (8) & Auditing/follow-up; reminders; rounding with staff for questions \\
\hline & Leader impact (7) & Leader engagement/support; SBIRT considered mandatory \\
\hline & Sustainment (3) & Problems anticipated ahead of time; new hire assimilation \\
\hline & Implementation efficiency (3) & Implementation planning phase; designated role (SC) \\
\hline \multirow{7}{*}{$\begin{array}{l}\text { Outcomes identified related to SBIRT implementation } \\
\text { were: }\end{array}$} & Awareness (13) & Clinician and patient awareness of risky substance use \\
\hline & Action-oriented process (11) & Ability to act on positive screen; more referral options accessible \\
\hline & Enhanced care transitions (8) & $\begin{array}{l}\text { Streamline continuum of care; support collaborative } \\
\text { communication }\end{array}$ \\
\hline & Therapeutic relationships (6) & Show clinician care; determine patient readiness in the process \\
\hline & $\begin{array}{l}\text { Connection to disease process } \\
\text { (6) }\end{array}$ & $\begin{array}{l}\text { Applicability of BI to other conditions; prevention of alcohol } \\
\text { withdrawal }\end{array}$ \\
\hline & SC development (4) & Implementation skills; organizational resource navigation \\
\hline & Comfort (4) & Clinician and patient comfort discussing substance use \\
\hline
\end{tabular}

"Some nurses on the floor were kind of freaked out by a new process. Staff felt like they were getting thrown a curve (ball) adding onto the admission process and were feeling anxious. I had to keep reassuring staff that they were making it harder than it was."

\section{Theme 2: implementation facilitators}

Participants also described several factors or resources that enhanced the timely and seamless implementation of SBIRT. The most common facilitators were leveraging support from staff nurses and interprofessional team members on the unit, adapting the intervention to fit the organizational context, and training and available support for SCs. One participant, reflecting on facilitators to implementation, stated, "Site coordinator training, supporting training resources, including power points, were super helpful. We also were able to modify (training materials) to meet our facility needs." Another participant stated:

"Education and guidelines that were provided by the study team were helpful. The collaborative work that we did through the monthly calls helped me to make sure that I was on the right track by (making me) aware of things I needed to work on.”

\section{Theme 3: implementation outcomes}

Participants described the outcomes of SBIRT implementation for nurses. Benefits to nurses on the unit included increased awareness of substance use risks amongst their patients, the inclusion of an actionoriented approach for patients with risky substance use, 
and enhanced care transitions across clinicians and care settings. Nurses gained the confidence to engage actively with patients, leading to more trusting and therapeutic relationships. Ultimately, increased awareness and confidence helped nurses feel more prepared to intervene with patients with addiction. As one participant stated, "SBIRT raised education and awareness of the clinicians. We were able to provide better resources and identify patients needing support. Patients were falling through the cracks before." Another participant said

'I think it gave us more of an awareness of patients' backgrounds. Many times, we

do not get the whole history, and it was helpful to collect additional information about factors that could be contributing to poor health. The process gave us an opportunity to provide more resources based on data collected."

\section{Survey}

Eleven (79\%) of the participants completed the followup survey. Figure 1 shows the most highly rated implementation strategies. According to participants, the most helpful (i.e., effective) implementation strategies were purposely reexamining the implementation $(M=4.38 ; n$ =8), provide ongoing consultation $(M=4.13 ; n=8)$, audit and provide feedback $(M=4.1 ; n=10)$, develop education materials $(M=4.1 ; n=10)$, identify and prepare champions $(M=4 ; n=7)$, and tailor strategies $(M=4 ; n=7)$.

\section{Discussion}

Acute care clinicians need to be prepared to use clinical interventions like SBIRT considering the increase in substance use and abuse; however, use in acute care units is limited. Using the framework provided by Powell et al. [14], this study examined SCs' perceptions of commonly used and helpful strategies for implementing SBIRT into acute care units. In the parent study, SCs were highly engaged in the SBIRT implementation and thus were able to provide insight into perceived challenges and successes of implementation. Based on the interviews, SCs identified barriers (e.g., challenges sustaining the implementation of SBIRT), facilitators (e.g., leveraging support from interprofessional team members), and outcomes (e.g., increased awareness of substance use risk) of SBIRT implementation. Among the implementation strategies, participants perceived that purposely reexamining the implementation, providing ongoing consultation, and auditing and providing feedback were the most helpful. Our study findings suggest that, although SCs experienced barriers to the implementation of SBIRT, the outcomes associated with SBIRT provided benefits to nurses and include increased awareness of

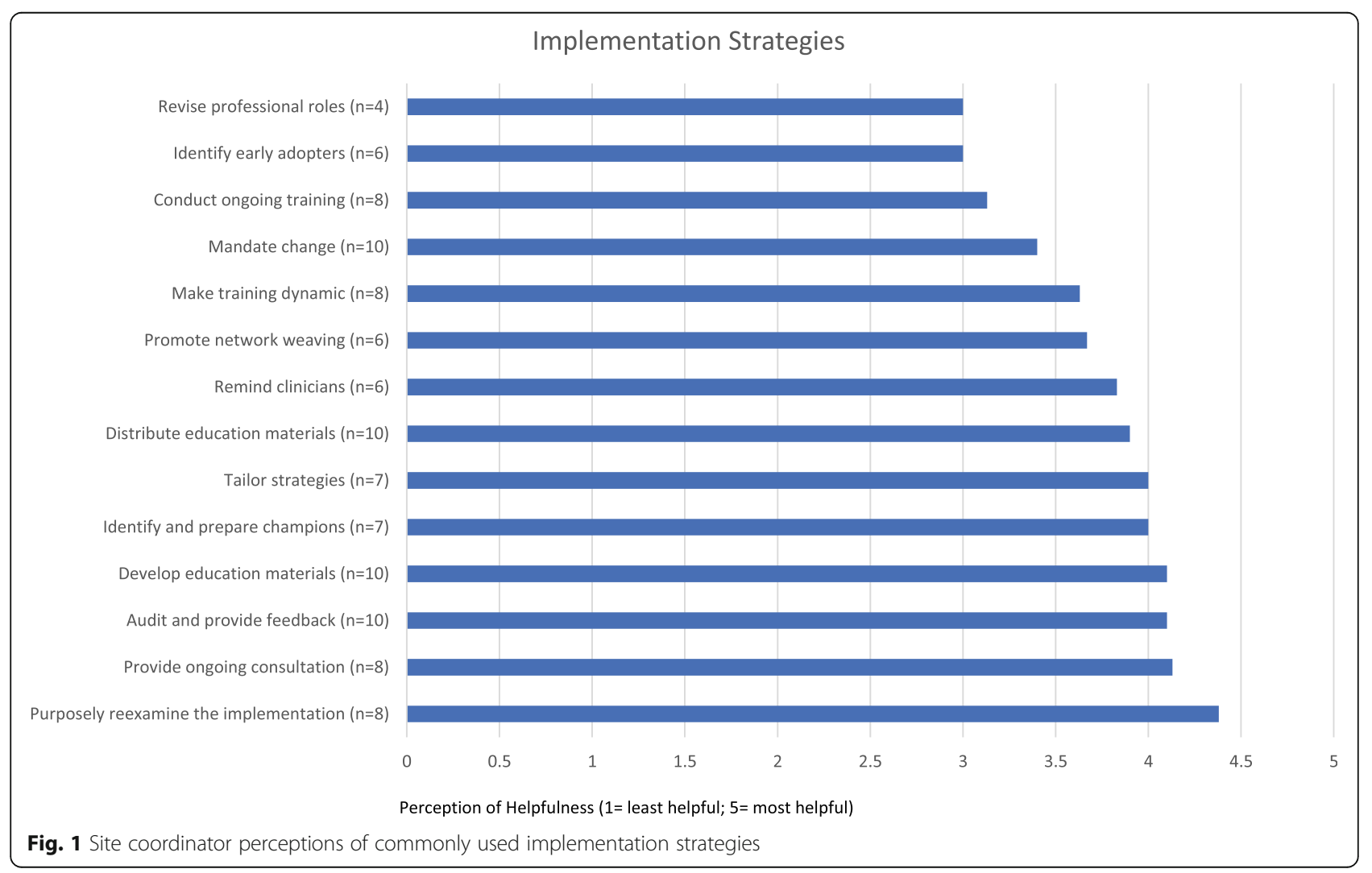


and confidence in addressing substance use and potential to positively impact overall treatment for patients with substance use.

Several SCs were new to implementation. However, they expressed that many implementation strategies helped enhance the implementation process. Our findings are consistent with other SBIRT implementation research studies that used implementation strategies as defined by Powell et al. [14], including audit and provide feedback $[8,21,22]$, develop education materials [21, 23], and tailor strategies [24]. Two strategies, audit and provide feedback, and tailor strategies, were rated among the most helpful and commonly used, similar to findings in a review of utilized implementation strategies [15]. All SCs voiced the value of available training resources, study team mentorship, and peer support as they moved through the implementation process. Given their experience, many SCs stated that they felt more confident leading change in the future due to the knowledge and skills they developed during SBIRT implementation.

SCs reported that it was important to leverage support from other interprofessional team members, such as social workers and clinical educators. Specifically, they noted the opportunity to identify additional stakeholders (e.g., unit secretary, peer coaches) and better align existing resources in preparation for SBIRT implementation. Many of the SCs had not implemented or used SBIRT, nor were they able to identify who delivered brief interventions or referral to treatment when needed. In follow up, site interviews were initiated to identify who delivered each phase of the clinical intervention [18]. Challenges with identifying stakeholders and aligning resources also might have been due to some of the SCs' limited leadership experiences. SCs may not have been aware of all of the resources available at their facilities or had experience leading interdisciplinary work. In the future, training should include exercises that help SCs to think through the SBIRT process and identify stakeholders as a group. These exercises may facilitate a deeper understanding of the stakeholders involved in SBIRT implementation and more comprehensive stakeholder engagement.

SCs reported that the sustainability of the SBIRT intervention was one of the primary barriers. Sustainability has been described as the ongoing use and evolution of a practice change within an environment over time [25]. According to SCs, integrating a new change into practice was challenging. SCs sensed process complacency as the study started to wind down, which some described as common with newly implemented change. As the study ended, the next steps for SBIRT integration and spread throughout the system were unclear. With the absence of structure previously provided by the study team,
SBIRT practice was difficult to sustain. For example, monthly meetings with the study team and routine study-related data collection activities motivated SCs to continue monitoring SBIRT implementation. Planning and measurement of sustainability are complex, with many not understanding the difference and the overlap between implementation and sustainability [25]. The lessons learned from implementing SBIRT included the importance of adapting clinical interventions to the environment and identifying expert stakeholders to partner with during the implementation process. However, an opportunity exists for strategic sustainability planning in early study phases to ensure that the clinical practice continues. While this study identified the perceived helpfulness of multiple implementation strategies, a systematic investigation is needed to understand why specific implementation strategies are helpful to change leaders.

\section{Limitations}

Limitations of this study include the small sample size, participant bias, and the interview approach. This study involved interviews from a small sample of SCs; albeit this is typical of quality improvement feedback when implementing changes within health systems. The respondents were all SCs and received similar training, which potentially biased responses to similar implementation processes. Interviewing additional staff members responsible for intervention delivery may have led to the identification of other themes or provided a more comprehensive identification of experiences and feedback related to the SBIRT implementation. Interviews were conducted over the telephone or e-mail and were not audio-recorded. Consequently, we were not able to capture some nonverbal data that may have contributed to the analysis, and we were limited to field notes collected during the interviews. Additionally, the semistructured approach used in the interviews may have limited generation of additional data related to SBIRT implementation.

\section{Conclusion}

SCs who led implementation efforts within a large healthcare system identified several barriers and facilitators to the implementation of SBIRT. Additionally, they identified nurse-related outcomes associated with the implementation of SBIRT into practice and strategies that were helpful in the implementation process. This information can inform the implementation of SBIRT and other interventions in acute care settings.

\section{Abbreviations}

ERIC: Expert Recommendations for Implementing Change; SBIRT: Screening, Brief Intervention, and Referral to Treatment; SC: Site coordinator 


\section{Acknowledgements}

The study team would like to acknowledge Laila Talib for providing manuscript edits and recommendations prior to submission.

\section{Authors' contributions}

AK performed data collection, analysis, and initial interpretation of the data. $K T$ verified the data analysis. AK, KT, and OU participated in the final interpretation and presentation of the data. RN conceptualized the manuscript scope and aims and provided senior author mentorship to the manuscript team. All authors provided major contributions to the manuscript writing, and read and approved the final manuscript.

\section{Funding}

This quality improvement study was part of a larger parent SBIRT study. Funding was provided with support from the Indiana University Health Values Fund Grand Challenge grant and the Indiana Clinical and Translational Sciences Institute funded in part by Grant \# UL1TR001108 from the National Institutes of Health, National Center for Advancing Translational Sciences, Clinical and Translational Sciences Award.

\section{Availability of data and materials}

The data generated and analyzed during the study are available from the corresponding author upon reasonable request.

\section{Declarations}

\section{Ethics approval and consent to participate}

The parent study was administratively reviewed by the Indiana University Institutional Review Board and determined not to be human subject research \#1801646970.

\section{Consent for publication}

Not applicable.

\section{Competing interests}

The authors declare that they have no competing interests. We confirm that the work is original and has not been published elsewhere, nor is it currently under consideration for publication elsewhere.

\section{Received: 18 August 2020 Accepted: 7 April 2021}

Published online: 23 April 2021

\section{References}

1. Substance Abuse and Mental Health Services Administration. Key substance use and mental health indicators in the United States: Results from the 2018 National Survey on Drug Use and Health. Vol. H-54, HHS Publication No. PEP19-5068, NSDUH Series H-54. Rockville; 2019. Available from: https:// www.samhsa.gov/data. Accessed 14 Mar 2020

2. National Institute on Drug Abuse. Health consequences of drug misuse. 2017. Available from: https://www.drugabuse.gov/related-topics/healthconsequences-drug-misuse. [cited 2019 Jun 29]

3. National Institute on Drug Abuse. Trends \& Statistics. 2017. Available from: https://www.drugabuse.gov/related-topics/trends-statistics. [cited 2018 Oct 10]

4. National Institute on Drug Abuse. Overdose death rates. 2019. Available from: https://www.drugabuse.gov/related-topics/trends-statistics/overdosedeath-rates. [cited 2020 Jan 16]

5. Brereton R, Gerdtz M. Alcohol and Other Drug (AOD) Education for hospital staff: an integrative literature review. Issues Ment Health Nurs. 2017;38(1):4260

6. Aldridge A, Linford R, Bray J. Substance use outcomes of patients served by a large US implementation of Screening, Brief Intervention and Referral to Treatment (SBIRT). Addiction. 2017;112(459 599):43-53.

7. Substance Abuse and Mental Health Services Administration. SBIRT: Screening, Brief Intervention, and Referral to Treatment. Available from: https://www.integration.samhsa.gov/clinical-practice/sbirt. [cited 2018 Sep 18]

8. Berger L, Hernandez-meier J, Hyatt J, Brondino M. Referral to treatment for hospitalized medical patients with an alcohol use disorder : A proof-ofconcept brief intervention study. Soc Work Health Care. 2017;56(5):321-34. https://doi.org/10.1080/00981389.2017.1299073.
9. Gormican EK, Hussein ZS. SBIRT (Screening, Brief Intervention, and Referral to Treatment) among trauma patients: A review of the inpatient process and patient experience. J Trauma Nurs. 2017;24(1):42-5.

10. Bray J, Cowell A, Hinde J. A systematic review and meta-analysis of health care utilization outcomes in alcohol screening and brief intervention trials. Med Care. 2011:47(3):287-94.

11. Madras BK, Compton WM, Avula D, Stegbauer T, Stein JB, Clark HW. Screening, brief interventions, referral to treatment (SBIRT) for illicit drug and alcohol use at multiple healthcare sites: Comparison at intake and 6 months later. Drug Alcohol Depend. 2009;99(1-3):280-95.

12. Désy PM, Howard PK, Perhats C, Li S. Alcohol screening, brief intervention, and referral to treatment conducted by emergency nurses: An impact evaluation. J Emerg Nurs. 2010;36(6):538-45. https://doi.org/10.1016/j.jen.2 009.09.011.

13. Braxter BJ, Puskar K, Mitchell AM, Hagle H, Gotham H, Terry MA. Nursing students' experiences with screening, brief intervention, and referral to treatment for substance use in the clinical/hospital setting. J Addict Nurs. 2014;25(3):122-9.

14. Powell BJ, Waltz TJ, Chinman MJ, Damschroder LJ, Smith JL, Matthieu MM et al. A refined compilation of implementation strategies: Results from the Expert Recommendations for Implementing Change (ERIC) project. Implement Sci. 2015:10(1):1-14

15. Powell BJ, Fernandez ME, Williams NJ, Aarons GA, Beidas RS, Lewis CC, et al. Enhancing the impact of implementation strategies in healthcare : a research agenda. Front Public Heal. 2019;7(3):1-9.

16. Newhouse R, Janney M, Gilbert A, Agley J, Bakoyannis G, Ferren M, et al. Study protocol testing toolkit versus usual care for implementation of screening, brief intervention, referral to treatment in hospitals : a phased cluster randomized approach. Addict Sci Clin Pract. 2018;13(28):1-10. https://doi.org/10.1186/s13722-018-0130-4.

17. Schwindt R, Agley J, Newhouse R, Ferren M. Screening, brief intervention and referral to treatment (SBIRT) training for nurses in acute care settings: lessons learned. Appl Nurs Res. 2019:48:19-21. https://doi.org/10.1016/j.a pnr.2019.05.014

18. Keen A, Thoele K, Newhouse R. Variation in SBIRT delivery among acute care facilities. Nurs Outlook. 2019;68(2):1-7.

19. Ogrinc G, Davies L, Goodman D, Batalden P, Davidoff F, Stevens D. SQUIRE 2.0 (Standards for QUality Improvement Reporting Excellence): Revised publication guidelines from a detailed consensus process. BMJ Qual Saf. 2016:25:986-92

20. Elo S, Kääriäinen M, Kanste O, Pölkki T, Utriainen K, Kyngas H. Qualitative content analysis : a focus on trustworthiness. SAGE Open. 2014;1-10.

21. Bacidore V, Letizia M, Mitchel AM. Implementing Interprofessional Alcohol Screening, Brief Intervention, and Referral to Treatment in the Emergency Department. Adv Emerg Nurs J. 2017;39(3):199-216 Available from: http:// insights.ovid.com/crossref?an=01261775-201707000-00007.

22. Bogenschutz MP, Donovan DM, Adinoff B, Crandall C, Forcehimes AA, Lindblad R, et al. Design of NIDA CTN Protocol 0047 : Screening, Motivational Assessment, Referral, and Treatment in Emergency Departments ( SMART-ED). Am J Drug Alcohol Abuse. 2011:37:417-25.

23. Broyles LM, Kraemer KL, Kengor C, Gordon AJ. A tailored curriculum of alcohol screening, brief intervention, and referral to treatment (SBIRT) for nurses in inpatient settings. J Addict Nurs. 2013;24(3):130-41.

24. Kaiser DJ, Karuntzos G. An Examination of the workflow processes of the Screening, Brief Intervention, and Referral to Treatment (SBIRT) Program in Health Care Settings. J Subst Abuse Treat. 2016;60(2016):21-6. https://doi. org/10.1016/j.jsat.2015.08.001

25. Penno LN, Davies B, Graham ID, Backman C, Macdonald I, Bain J, et al. Identifying relevant concepts and factors for the sustainability of evidencebased practices within acute care contexts : a systematic review and theory analysis of selected sustainability frameworks. Implement Sci. 2019;14(108): $1-16$.

\section{Publisher's Note}

Springer Nature remains neutral with regard to jurisdictional claims in published maps and institutional affiliations. 